\title{
NONUNIQUENESS OF EXTREMAL KERNELS ${ }^{1}$
}

\author{
JAMES G. CAUGHRAN
}

Given a "kernel" $k=k\left(e^{i \theta}\right) \in L^{q}$, then

$$
\Phi(f)=\frac{1}{2 \pi i} \int_{|z|=1} f(z) k(z) d z
$$

is a bounded linear functional on $H^{p}(1 / p+1 / q=1)$ with norm $\|\Phi\| \leqq\|k\|_{\alpha}$. Two kernels induce the same linear functional, $k_{1} \sim k_{2}$, if and only if they differ by an $H^{q}$ function. It can be shown that the duality relation, $\|\Phi\|=\inf \left\{\left\|k_{1}\right\|_{q}: k_{1} \sim k_{2}\right\}$ holds for $1 \leqq p \leqq \infty$. One may ask whether an extremal kernel exists, i.e., a kernel $k_{1} \sim k$ such that $\|\Phi\|=\left\|k_{1}\right\|_{q}$, and if so, whether it is unique.

By functional analytic methods, Havinson [1] and Rogosinski and Shapiro [2] showed that an extremal kernel always exists for $1 \leqq p$ $\leqq \infty$ and is unique for $1<p \leqq \infty$. However, Rogosinski and Shapiro constructed a counterexample to show that it need not be unique for $p=1$. As their example to show nonuniqueness for $p=1$ was rather complicated, we present a simplification of their example.

Let

$$
\begin{aligned}
& k\left(e^{i \theta}\right)=1, \quad 0 \leqq \theta<\pi / 2, \\
& =-1, \quad \pi / 2 \leqq \theta<\pi, \\
& =0, \quad \pi \leqq \theta<2 \pi \text {. }
\end{aligned}
$$

Rogosinski and Shapiro showed that this kernel induces a functional $\Phi$ on $H^{1}$ with $\|\Phi\|=1$, and thus $k$ is an extremal kernel. To show this extremal kernel is not unique, it suffices to produce an $H^{\infty}$ function $h \neq 0$ for which $\|k+h\|_{\infty}=1$.

Let $h$ be the conformal mapping sending the unit disk to the upper half-disk $|w|<1$, Im $w>0$, with $h(1)=-1, h(i)=0$, and $h(-1)=1$. Then $h \in H^{\infty}$, and

$$
\begin{aligned}
& -1 \leqq h\left(e^{i \theta}\right)<0, \quad 0 \leqq \theta<\pi / 2 \\
& 0 \leqq h\left(e^{i \theta}\right)<1, \quad \pi / 2 \leqq \theta<\pi \\
& \left|h\left(e^{i \theta}\right)\right|=1, \quad \pi \leqq \theta<2 \pi .
\end{aligned}
$$

Thus $\left|k\left(e^{i \theta}\right)+h\left(e^{i \theta}\right)\right| \leqq 1$, and $h$ is the desired function.

Received by the editors December $8,1966$.

1 This research was supported in part by a National Science Foundation Grant. The author would like to thank Professor P. L. Duren, who suggested the problem in a course at the University of Michigan. 


\section{REFERENCES}

1. S. Ya. Havinson, On some extremal problems in the theory of analytic functions, Amer. Math. Soc. Transl. (2) 32 (1963), 139-154.

2. W. W. Rogosinski and H. S. Shapiro, On certain extremum problems for analytic functions, Acta Math. 90 (1953), 287-318.

UNIVERSITY OF MichigaN

\section{POSITIVE $H^{1 / 2}$ FUNCTIONS ARE CONSTANTS}

\section{J. NEUWIRTH AND D. J. NEWMAN}

The Koebe function $z /(1+z)^{2}$ is positive everywhere on $|z|=1$, $z \neq-1$, and lies in the Hardy class $H^{p}$ for every $p<1 / 2$. We show that this behavior is extreme by proving the following

THEOREM. If $f(z) \in H^{1 / 2}$ and if $f(z) \geqq 0$ a.e. on $|z|=1$ then $f(z)$ is a constant.

Proof. We may assume that $f(z)$ is not identically 0 . If $B(z)$ denotes the Blaschke product for the zeros of $f(z)$ then, as usual, we can write

$$
f(z)=B(z) F^{2}(z), \quad F(z) \in H^{1} .
$$

We write the condition $f(z) \geqq 0$ as the equation $f(z)=|f(z)|$ and conclude from (1) that

$$
B(z) F^{2}(z)=\left|F^{2}(z)\right| \text { a.e. on }|z|=1 .
$$

Since $f(z)$ is not identically 0 it follows that $F(z)$ is nonzero a.e. on $|z|=1$. Thus we may divide (2) by $F(z)$ and obtain

$$
B(z) F(z)=\overline{F(z)} \quad \text { a.e. on }|z|=1 \text {. }
$$

But the left side of (3) is $H^{1}$ and so has all negative Fourier coefficients 0 , the right side is conjugate $H^{1}$ and so has all positive Fourier coefficients 0 !.

Thus only the constant term remains and we conclude that both sides are constants. This is to say $B(z) F(z)$ and $F(z)$ are both constants and so indeed $f(z)=(B(z) F(z)) . F(z)$ is a constant.

University OF CONNECTICUT AND Yeshiva University

Received by the editors May 17, 1967. 